Page 1 of $2 \quad$ Proj.

\begin{tabular}{|c|c|c|c|c|c|}
\hline \multirow{3}{*}{\multicolumn{2}{|c|}{ 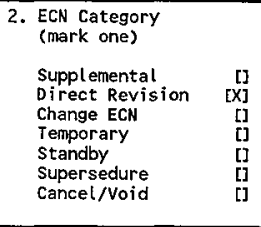 }} & \multicolumn{2}{|c|}{$\begin{array}{l}\text { 3. Originator's Name, Organization, MSIN, } \\
\text { and Tel ephone No. } \\
\text { DL Ke } 71 \mathrm{y} / 08000 / \mathrm{H} 1-15 / 376-2276\end{array}$} & 4. USQ Required? & $\begin{array}{l}\text { 5. Date } \\
10 / 27 / 98 \\
\end{array}$ \\
\hline & & \multicolumn{2}{|c|}{$\begin{array}{l}\text { 6. Project Title/No./Work Order No. } \\
\text { SARP Upgrade P1an }(772027 / 108)\end{array}$} & $\begin{array}{l}\text { 7. Bldg./Sys./Fac. No. } \\
\text { NA }\end{array}$ & $\begin{array}{l}\text { 8. Approval Designator } \\
\text { NA }\end{array}$ \\
\hline & & \multicolumn{2}{|c|}{$\begin{array}{l}\text { 9. Document Numbers Changed by this ECN } \\
\text { (includes sheet no. and rev.) } \\
\text { HNF-SD-TP-PLN-006, Rev. } 5\end{array}$} & $\begin{array}{l}\text { 10. Related ECN No(s). } \\
\text { NA }\end{array}$ & $\begin{array}{l}\text { 11. Related PO No. } \\
\text { NA }\end{array}$ \\
\hline \multirow{2}{*}{\multicolumn{2}{|c|}{$\begin{array}{l}\text { 12a. Modification Work } \\
\text { [] Yes (fill out Blk. } \\
12 b \text { ) } \\
{[X] \text { No (NA Blks. 12b, }} \\
12 \mathrm{c}, 12 \mathrm{~d} \text { ) }\end{array}$}} & \multirow[t]{2}{*}{$\begin{array}{l}\text { 12b. Work Package } \\
\text { No. } \\
\text { NA }\end{array}$} & $\begin{array}{l}\text { 12c. Modification work Complete } \\
\text { NA }\end{array}$ & \multicolumn{2}{|c|}{$\begin{array}{l}\text { 12d. Restored to Original Condi- } \\
\text { tion (Temp. or Standby ECN only) } \\
\text { NA }\end{array}$} \\
\hline & & & $\begin{array}{l}\text { Design Authority/Cog. Engineer } \\
\text { Signature \& Date }\end{array}$ & \multicolumn{2}{|c|}{$\begin{array}{l}\text { Design Authority/Cog. Engineer } \\
\text { Signature \& Date }\end{array}$} \\
\hline \multicolumn{6}{|c|}{$\begin{array}{l}\text { 13a. Description of change } \\
\text { This is a complete revision. This revision to the Safety Analys is Report for Packaging } \\
\text { (SARP) Upgrade Plan reflects a revised SARP Upgrade schedule based on the most current } \\
\text { program needs. References have been updated. }\end{array}$} \\
\hline
\end{tabular}

14a. Justification (mark one)

\begin{tabular}{|lllllll}
$\begin{array}{lll}\text { Criteria Change } \\
\text { As-Found }\end{array}$ & {$[X]$} & Design Improvement & {[]} & Environmental & {[]} & Facility Deactivation \\
\hline & {[]} & Facilitate Const & {[]} & Const. Error/omission & [] & Design Error/Omission \\
\hline
\end{tabular}

14b. Justification Details

Changes to the SARP Upgrade PIan reflect current SARP needs for the Hanford Site.

15. Distribution (include name, MSIN, and no. of copies)

See attached.
RELEASE STAMP

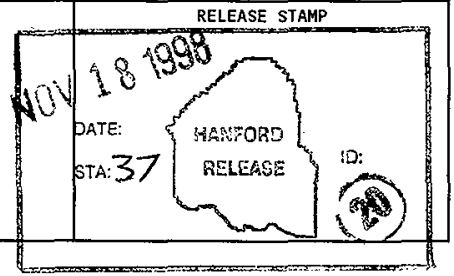




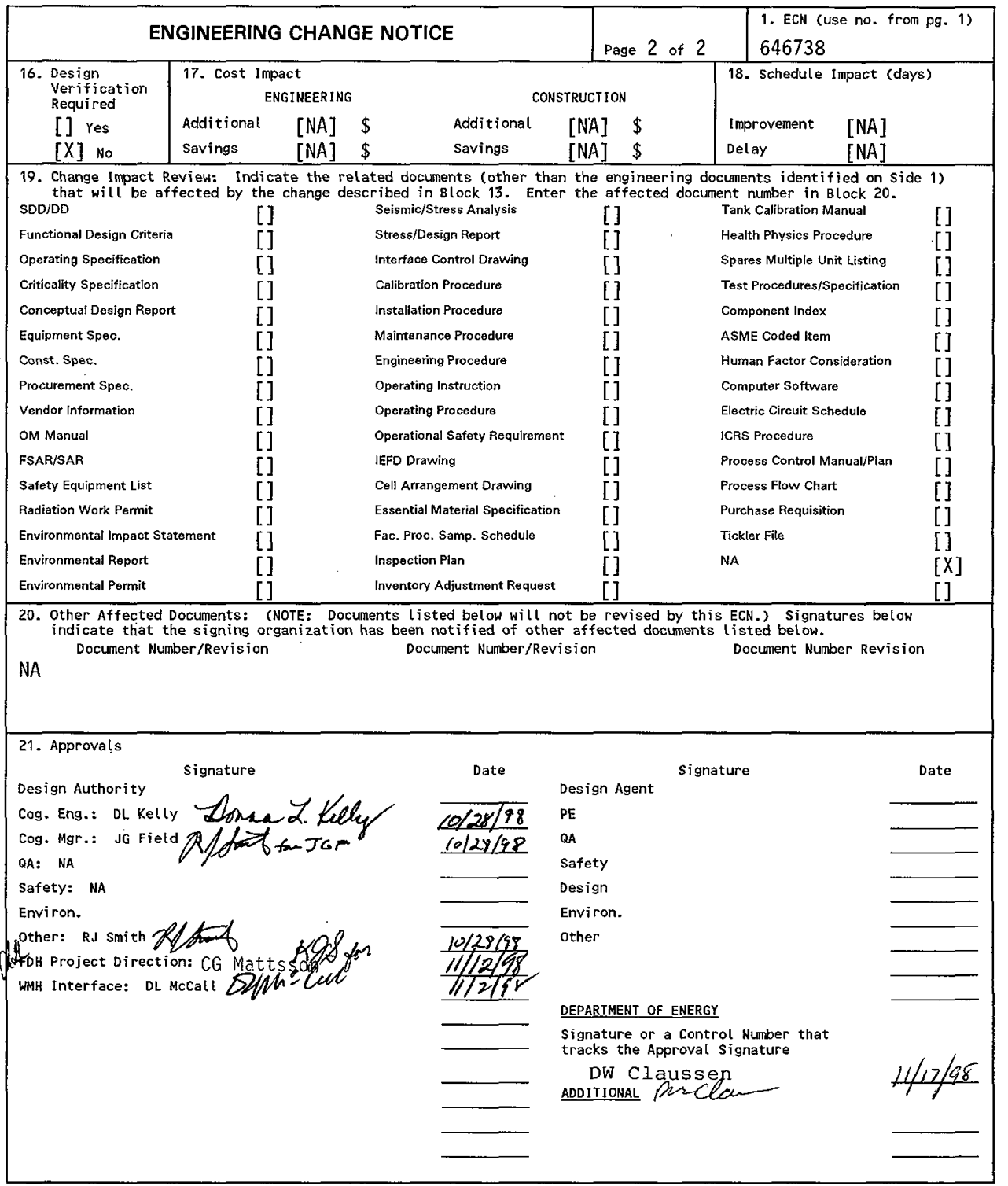




\title{
Safety Analysis Report for Packaging Upgrade Plan
}

\author{
D. L. Kelly
}

Waste Management Federal Services, Inc., Northwest Operations, Rich T and, WA 99352

U.S. Department of Energy Contract DE-AC06-96RL13200

$\begin{array}{ll}\text { EDT/ECN: EOf } 646738 & \text { UC: } 513 \\ \text { Org Code: } 08000 & \text { Charge Code: } 772027 / 108 \\ \text { B\&R Code: N/A } & \text { Total Pages: } 11\end{array}$

Key Words: Safety Analysis Report for Packaging, SARP, upgrade plan, performance agreement, performance expectation

Abstract: This Safety Analysis Report for Packaging (SARP) Upgrade Plan reflects a revised SARP upgrade schedule based on the most current program needs. A Project Hanford Management Contract (PHMC) Performance Expectation exists to update, revise, and/or cancel seven onsite SARPs during FY 1999. It is the U.S. Department of Energy's desire that $100 \%$ of the SARPs (which existed at the beginning of the PHMC Contract) be upgraded, revised, and/or canceled by the end of the five year contract. This plan is a "living" document and is used as a management tool.

TRADEMARK DISCLAIMER. Reference herein to any specific commercial product, process, or service by trade name, trademark, manufacturer, or otherwise, does not necessarily constitute or imply its endorsement, recommendation, or favoring by the United States Government or any agency thereof or its contractors or subcontractors.

Printed in the United States of America. To obtain copies of this document, contact: Document Control Services, P.0. Box 950, Mailstop H6-08, Richland WA 99352, Phone (509) 372-2420;

Fax (509) 376-4989.

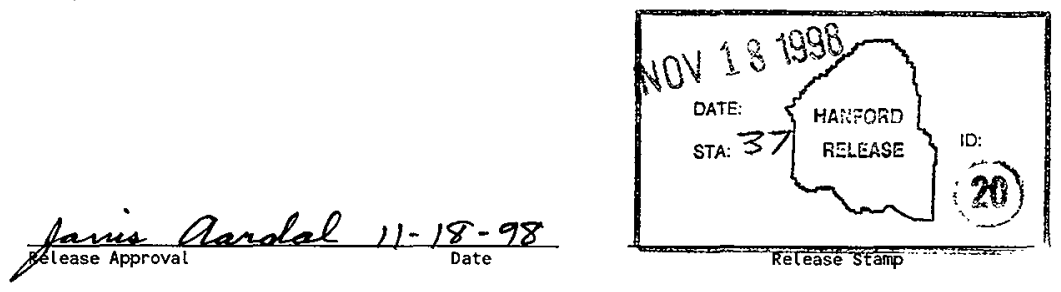

Approved for Public Release 
(2) Title

SAFETY ANALYSIS REPORT FOR PACKAGING UPGRADE PLAN

CHAHGE COMTROL RECORO

(3) Revision (4) Deseription of Change - Replace, Add, and Delate Pages

\begin{tabular}{l|l|l}
0 & $(7)$ EDT $142682,7 / 14 / 93$
\end{tabular}

O-A $\quad$ ECN 196183, 11/2/93

$1 \quad$ ECN $606657,5 / 24 / 94$

$2: \quad$ ECN $621440,8 / 14 / 95$

$3 \quad$ ECN $637762,12 / 7 / 96$

$4 \quad$ ECN $637778,9 / 2 / 97$

5. RS This is a complete revision. This revision reflects a SARP upgrade schedule based on the mose current program needs. References have been updated. Per ECN 640857.

6 RS

This revision reflects a SARP upgrade schedule based on the most current program needs. References have been updated. Replace all pages. Per ECN 64.6738.
Authorized for Release

(5) Cog. Engr. (6) Cog. Mgr. Date
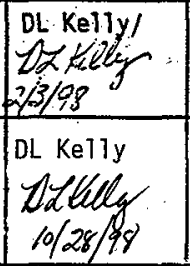

JG Field/ 20 JG Field

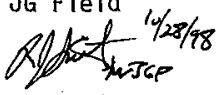


HNF-SD-TP-PLN-006 Rev. 6

\section{TABLE OF CONTENTS}

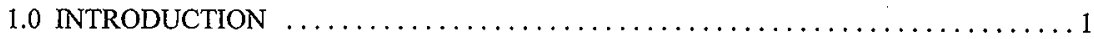

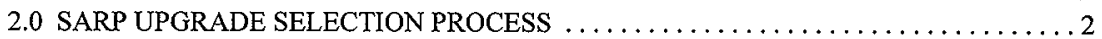

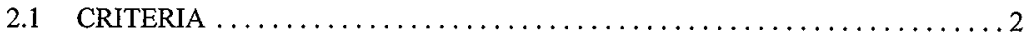

2.2 PLANNED SARP UPGRADES AND CANCELLATIONS $\ldots \ldots \ldots \ldots \ldots \ldots$

3.0 SUMMARY AND RECOMMENDATIONS $\ldots \ldots \ldots \ldots \ldots \ldots \ldots \ldots \ldots \ldots \ldots$

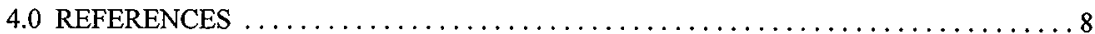

\section{LIST OF TABLES}

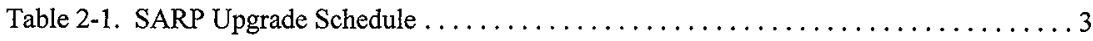


HNF-SD-TP-PLN-006 Rev. 6

\section{SAFETY ANALYSIS REPORT FOR PACKAGING UPGRADE PLAN}

\subsection{INTRODUCTION}

The purpose of this report is to provide an upgrade plan for all existing Safety Analysis Reports for Packaging (SARP) (onsite). This plan will also include the addition of new SARPs to the SARP completion schedule.

About half of the existing approved SARPs in use at Hanford are over ten years old, and were originally written and approved by several different contractors. Therefore, the packaging approval criteria, approval authorities, and method of documentation are not consistent among the SARPs. These issues were noted as a finding in a 1992 U.S. Department of Energy, Richland Operations Office (DOE/RL) Surveillance (Fisher 1992).

Various actions have been taken over the last several years to upgrade existing SARPs, cancel unused or inadequate SARPs, and to develop the technical safety criteria for onsite packagings. Also, existing approved SARPs have been entered into a copy control process to better control and track their use. These actions, in conjunction with this SARP Upgrade Plan, have resulted in higher quality SARPs that are consistent in their technical approach.

A Performance Agreement (WM5.1.1) was assigned, beginning in fiscal year 1997 (FY 1997), to update, revise, and/or cancel 20 percent of the existing onsite SARPs (as of FY 1997), so that 100 percent are reviewed and within current standards by the completion of the Project Hanford Management Contract (PHMC). This contract is for a five-year period, beginning in FY 1997 and ending in FY 2001. In FY 1998, Performance Agreement WM5.1.1 was identified as a Performance Expectation to allow the PHMC to measure performance based on the overall ability of Waste Management of Hanford, Inc. (WMH), to complete listed activities and milestones due to changing budgets, priorities, and DOE/RL direction.

As identified within HNF-PRO-154, Responsibilities and Procedures for All Hazardous Material Shipments, safety analysis documentation is reviewed, approved, and revised according to HNF-PRO-705, Safety Basis Planning, Documentation, Review and Approval. All onsite SARPs shall be reviewed and updated as necessary, at least every five years, and shall have a controlled distribution. Existing SARPs are grand fathered based on their original safety basis/authorization for up to five years, based on this SARP Upgrade Plan. The SARPS identified within this upgrade plan that are pre 1987 typically have a SARP identification number of "SAP" or "UNI," and require immediate attention. 
HNF-SD-TP-PLN-006 Rev. 6

\subsection{SARP UPGRADE SELECTION PROCESS}

The following describes the criteria and implementation of the SARP Upgrade Plan. The plan will be revised periodically (at least annually) to provide direction for SARP improvements, ensure compliance with HNF-PRO-154, Responsibilities and Procedures for All Hazardous Material Shipments, and to reflect the PHMC performance agreement requirements.

Early in FY 1997, thirty-four SARPs were identified for upgrade or cancellation. During FY 1997, a facility transfer from the Pacific Northwest National Laboratory (PNNL) to the PHMC occurred. As a result of this transfer, six SARPs were added into the SARP Upgrade Plan, and identified for upgrade in FY 1999. Subsequently, Revision 4 to the SARP Upgrade Plan (WHC 1997) identified a total of forty SARPs to be upgraded or canceled over the identified five-year period.

Management review in FY 1998 determined that three of the six new SARPs slated for upgrade in FY 1999 should be removed from the SARP Upgrade Plan as it was determined that SEP documents for these three were not needed. Thirty-seven SARPS are now identified for upgrade between FY 1997 and FY 2001. The seven SARPs that were identified for upgrade or cancellation during FY 1997 were completed. Eight SARPs have been targeted for upgrade during FY 1998, and it has been determined that seven to eight SARPs would need to be targeted in the out years so that the requirements identified within HNF-PRO-154, Responsibilities and Procedures for All Hazardous Material Shipments, and the identified performance agreement may be achieved.

The completed and anticipated SARP upgrades and cancellations over the identified fiveyear period are shown in Table 2-1 of this plan.

\subsection{CRITERIA}

Ranking of the current SARPs shown in Table 2-1 is based on either the age of the SARP, or on a combination of the age of the SARP and known funding to be in place for upgrade of the SARP. The older SARPs are targeted for upgrade prior to a newer SARP. If funding has been authorized by a client for upgrade of a SARP, that SARP is targeted for upgrade prior to another.

Safety analysis documentation is reviewed and updated as necessary, at least every five years, and has a controlled distribution. Existing SARPs are grand fathered based on their original safety basis/authorization for up to five years, based on this SARP Upgrade Plan. The SARPs identified within this upgrade plan that are pre 1987 typically have a SARP identification number of "SAP" or "UNI," and require immediate attention. 
Currently, thirty-seven SARPs are identified for upgrade between FY 1997 and FY 2001. The identification of SARPs to be upgraded in FY 2002 is being noted because of the five-year requirement for review and upgrade that is identified within HNF-PRO-154, Responsibilities and Procedures for All Hazardous Material Shipments.

\subsection{PLANNED SARP UPGRADES AND CANCELLATIONS}

A schedule of SARP upgrades and SARP cancellations through FY 2002 is depicted in Table 2-1. The status of the upgrade or cancellation process, as it applies to the performance agreement (FY 1997 through FY 2001), is also shown in Table 2-1.

Table 2-1. SARP Upgrade Schedule (5 pages)

\begin{tabular}{|c|c|c|c|c|c|}
\hline \multicolumn{6}{|c|}{ PY 1997 SARP Upgrades } \\
\hline SARPNumber and & $\begin{array}{l}\text { Cost } \\
\text { Complete } \\
\text { Review }\end{array}$ & Expires & Tite. & Planstatis & Ssers \\
\hline $\begin{array}{l}\text { SPRP SAP } 013 \\
\text { ReV } 6 \text { P }\end{array}$ & $\begin{array}{l}5 / 87 \\
\mathrm{ReV}, 2 \\
\mathrm{ECNN} / \mathrm{A}\end{array}$ & 180 & $\begin{array}{l}\text { Rairoad Liqud Waste Tanik } \\
\text { Cars }\end{array}$ & $\begin{array}{l}\text { Canceled } \\
9 / 2 \% 7 \% \text { ? } \\
\text { eCN } 642854\end{array}$ & byo \\
\hline $\begin{array}{l}\mathrm{SPRE} \\
\mathrm{ReVP}\end{array}$ & $09 / 23 / 82$ & $\mathrm{TBD}$ & Iradjated Fuel Cask Cars & $\begin{array}{l}\text { Ganceled } \\
9 / 2 / 97 \\
\mathrm{ECN} 642855\end{array}$ & DESH DMN \\
\hline $\begin{array}{l}\mathrm{SPRE}-\mathrm{SAP} 092 \\
\mathrm{ReV} 2\end{array}$ & $\begin{array}{l}12 / 13 / 88 \\
\text { Revo } 0 \text { मे }\end{array}$ & TBD & 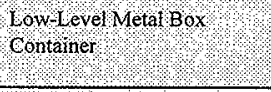 & Canceled & $\mathrm{HWH}^{\mathrm{NH}}$ \\
\hline 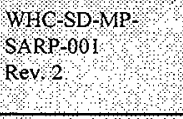 & Rev1 11992 & 1997 & $\begin{array}{l}\text { Boorstop Sanple Carriet } \\
\text { System } \\
\text { (replaced by HNF-SD-TP } \\
\text { SARP-023) }\end{array}$ & $\begin{array}{l}\text { Upgraded } \\
2 \% 24 / 97 \\
\text { EeN } 619550\end{array}$ & HMe NHC \\
\hline $\mathrm{SP}-\mathrm{RE} \mathrm{SAP} 043$ & $03705 / 85$ & $\mathrm{TBD}$ & Whternally Shielded $5 \mathrm{~s} g$ al & $\begin{array}{l}\text { Canceted } \\
4 / 28 / 97 \\
\mathrm{ECN} 637780\end{array}$ & PNNL \\
\hline $\begin{array}{l}\text { SD RE-SAP } 083 \\
\text { Rev } 1\end{array}$ & Rev 0 & $\mathrm{TBD}$ & $\mathrm{AL}-\mathrm{M} 6, \mathrm{CoC}$ & $\begin{array}{l}\text { Canceled } \\
\text { S/9/97 } \\
\text { ECN } 637784\end{array}$ & $B W H C$ \\
\hline $\begin{array}{l}\text { SDRE SAP } 084 \\
\text { Rev } 1\end{array}$ & $\begin{array}{l}09 / 05 / 84 \\
\operatorname{Rev} 0\end{array}$ & $T B D$ & $21-P F 1$ Overpack/Zircalioy & $\begin{array}{l}\text { Canceled } \\
4 / 2867 \\
\text { ECN } 639781\end{array}$ & BWHC: \\
\hline
\end{tabular}


Table 2-1. SARP Upgrade Schedule (5 pages)

\begin{tabular}{|c|c|c|c|c|c|}
\hline \multicolumn{6}{|c|}{ ( } \\
\hline $\begin{array}{l}\text { SARP Number and } \\
\text { Curent Revision }\end{array}$ & $\begin{array}{l}\text { Last } \\
\text { Complete } \\
\text { Review }\end{array}$ & Expres & Titte & Planistatus & Osers \\
\hline $\mathrm{SPRE} \mathrm{RAP} 025$ & $\begin{array}{l}03 / 01 / 84 \\
\mathrm{ReV} 0 \\
\mathrm{EDTN} / \mathrm{A}\end{array}$ & सBD & $\begin{array}{l}\text { Decontaminated Equipment } \\
\text { Self Container }\end{array}$ & $\begin{array}{l}\text { Bpgraded } \\
9 / 29 / 98 \\
\mathrm{LDT} 622968\end{array}$ & WMV BW\%s; \\
\hline $\begin{array}{l}\mathrm{SBrRE}-\mathrm{SAP}-044 \\
\mathrm{Rev} / \mathrm{B}\end{array}$ & $07 / 06 / 84$ & $\mathrm{ABD}$ & $225 \mathrm{~B}$ waste Cask & $\begin{array}{l}\text { Ganceled } \\
910 / 98 \\
\mathrm{ESN} 642865\end{array}$ & BWHC \\
\hline 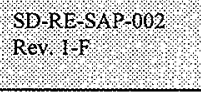 & $02 / 12 / 82$ & TBB & $\begin{array}{l}\text { TRU Contaminated liquid } \\
\text { organics }\end{array}$ & $\begin{array}{l}\text { Upgraded } \\
\text { O/30\%98 } \\
\mathrm{EDT} 622967\end{array}$ & WMH. $\mathrm{WWHC}$ \\
\hline 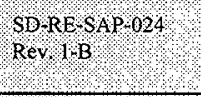 & $09 / 22 / 83$ & सBD & Non-TRU, Non fissile Waste, & $\begin{array}{l}\text { Upgraded } \\
9 / 30 / 98 \\
\mathrm{EBT} 622967\end{array}$ & WMH, BWHC, \\
\hline $\begin{array}{l}\mathrm{SPRP} S \mathrm{SAP} P 33 \\
\mathrm{ReV}\end{array}$ & $\begin{array}{l}06 / 26 / 84 \\
\mathrm{Rev} / \mathrm{EONN} / \mathrm{A}\end{array}$ & $\mathrm{TBO}$ & TRU 5 s gal Drun & $\begin{array}{l}\text { rupgraded } \\
9 / 30 / 88 \\
\mathrm{EDT} 622967\end{array}$ & WMir. BWHC, \\
\hline $\begin{array}{l}\mathrm{SDRESAP-091} \\
\mathrm{Rev} 1\end{array}$ & $\begin{array}{l}02 / 18 / 88 \\
\mathrm{Rev} 0\end{array}$ & TBD & TRU Metal Box Container & $\begin{array}{l}\text { canceled } \\
910 \% 98 \\
\mathrm{BON} 642864\end{array}$ & BWHe \\
\hline $\begin{array}{l}\mathrm{WHC}-\mathrm{SD}-\mathrm{NR} \\
\mathrm{SARP} 004 \\
\mathrm{ReV} / \mathrm{C}-\mathrm{l} \\
(\mathrm{UNI}-2849)\end{array}$ & $09 / 17 / 84$ & गBD & lon Exchange Modules & $\begin{array}{l}\text { Gpgraded } \\
912 \mathrm{~V} 98 \\
\text { EDT } 62295\end{array}$ & DESH \\
\hline WHC SD FF SARP & $\begin{array}{l}05 / 02 / 90 \\
\mathrm{Rev}\end{array}$ & $04 / 05 / 96$ & Radial Reflector Shipping & $\begin{array}{l}\text { Cancefed } \\
9 / 10 / 98 \\
\mathrm{ECN} / 642866\end{array}$ & BWHC \\
\hline \multicolumn{6}{|c|}{ 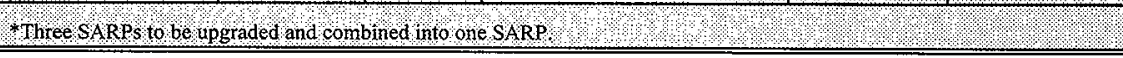 } \\
\hline \multicolumn{6}{|c|}{ FY 1999 SARP Upgrades } \\
\hline $\begin{array}{l}\text { SARP Number and } \\
\text { Current Revision }\end{array}$ & $\begin{array}{l}\text { Last } \\
\text { Complete } \\
\text { Review }\end{array}$ & Expires & Title & Plan/Status & Users \\
\hline $\begin{array}{l}\text { HNF-SD-TP-SEP- } \\
058 \text { Rev. (New) }\end{array}$ & $\begin{array}{l}x-f e r \text { from } \\
\text { PNNL to } \\
\text { BWHC in } \\
\text { FY } 97 \\
\end{array}$ & & SERF Cask SEP & $\begin{array}{l}\text { To be } \\
\text { Upgraded }\end{array}$ & BWHC \\
\hline $\begin{array}{l}\text { HNF-SD-TP-SEP. } \\
059 \text { Rev. (New) }\end{array}$ & $\begin{array}{l}x \text {-fer from } \\
\text { PNNL to } \\
\text { BWHC in } \\
\text { FY97 }\end{array}$ & & $\begin{array}{l}\text { Radioactive Waste Disposal } \\
\text { Cask SEP }\end{array}$ & $\begin{array}{l}\text { To be } \\
\text { Canceled }\end{array}$ & BWHC \\
\hline
\end{tabular}


Table 2-1. SARP Upgrade Schedule (5 pages)

\begin{tabular}{|c|c|c|c|c|c|}
\hline $\begin{array}{l}\text { HNF-SD-TP-SEP- } \\
063 \text { Rev. (New) }\end{array}$ & $\begin{array}{l}x \text {-fer from } \\
\text { PNNL to } \\
\text { BWHC in } \\
\text { FY97 } \\
\end{array}$ & & PRTR Cask SEP & $\begin{array}{l}\text { To be } \\
\text { Canceled }\end{array}$ & BWHC \\
\hline $\begin{array}{l}\text { WHC-SD-WM- } \\
\text { SARP-001 } \\
\text { Rev. 1-C }\end{array}$ & $\begin{array}{l}\text { 08/20/90 } \\
\text { Rev. } 0\end{array}$ & TBD & $\begin{array}{l}\text { Lead-Lined Drum/21-PF-1 } \\
\text { Packaging System }\end{array}$ & $\begin{array}{l}\text { To be } \\
\text { Upgraded }\end{array}$ & WMH, BWHC \\
\hline $\begin{array}{l}\text { WHC-SD-NR- } \\
\text { SARP-002 } \\
\text { Rev. 1-D }\end{array}$ & $\begin{array}{l}01 / 11 / 88 \\
\text { Rev. } 0 \\
\text { EDT } \\
\# 111016 \\
\end{array}$ & $06 / 01 / 96$ & OH-142 Mark I (Big Bertha) & $\begin{array}{l}\text { To be } \\
\text { Canceled }\end{array}$ & DESH \\
\hline $\begin{array}{l}\text { SD-RE-SAP-054 } \\
\text { Rev. 3-B }\end{array}$ & $\begin{array}{l}03 / 05 / 84 \\
\operatorname{Rev.} 2\end{array}$ & TBD & $\begin{array}{l}\text { 21-PF-1 Overpack/EBR-II } \\
\text { Spent Fuel Cask }\end{array}$ & $\begin{array}{l}\text { To be } \\
\text { Upgraded } \\
\text { (1/2 in FY } 98 \\
1 / 2 \text { in FY 99) } \\
\end{array}$ & WMH, BWHC \\
\hline $\begin{array}{l}\text { WHC-SD-NR- } \\
\text { SARP-001 } \\
\text { Rev. I-A }\end{array}$ & $\begin{array}{l}12 / 05 / 88 \\
\text { Rev. } 0 \\
\text { EDT } \\
\# 465716 \\
\end{array}$ & TBD & $\begin{array}{l}\text { Finished Fuel Assembly } \\
\text { Shipping Package }\end{array}$ & $\begin{array}{l}\text { To be } \\
\text { Canceled }\end{array}$ & BWHC \\
\hline \multicolumn{6}{|c|}{ FY 2000 SARP Upgrades } \\
\hline $\begin{array}{l}\text { SARP Number and } \\
\text { Current Revision }\end{array}$ & $\begin{array}{l}\text { Last } \\
\text { Complete } \\
\text { Review } \\
\end{array}$ & Expires & Title & Plan/Status & Users \\
\hline $\begin{array}{l}\text { WHC-SD-TP-SARP- } \\
005 \text { Rev. 0-C }\end{array}$ & $\begin{array}{l}\text { 09/20/94 } \\
\text { Rev. } 0 \\
\end{array}$ & $\mathrm{TBD}$ & $\begin{array}{l}\text { Modified Fuel Spacer Burial } \\
\text { Box }\end{array}$ & & $\begin{array}{l}\text { BWHC, WMH, } \\
\mathrm{BHI}\end{array}$ \\
\hline $\begin{array}{l}\text { WHC-SD-TP-SARP- } \\
006 \text { Rev. 0-A }\end{array}$ & $\begin{array}{l}04 / 19 / 94 \\
\text { Rev. } 0 \\
\end{array}$ & TBD & 85-Gai Retrieval Drum & & $\begin{array}{l}\text { WMH, DESH, } \\
\text { COGEMA }\end{array}$ \\
\hline $\begin{array}{l}\text { WHC-SD-TP-SARP. } \\
007 \text { Rev. 0-F }\end{array}$ & $\begin{array}{l}05 / 23 / 94 \\
\text { Rev. } 0 \\
\end{array}$ & TBD & Flexible Material Packaging & & $\begin{array}{l}\text { WMH, BWHC, } \\
\text { BHI, DESH }\end{array}$ \\
\hline $\begin{array}{l}\text { WHC-SD-TP-SARP- } \\
003 \text { Rev. 0-B }\end{array}$ & $\begin{array}{l}08 / 21 / 92 \\
\text { Rev. } 0\end{array}$ & TBD & Disposable Solid Waste Cask & & WMH, BWHC \\
\hline $\begin{array}{l}\text { WHC-SD-TP-SARP- } \\
004 \text { Rev. 0-B }\end{array}$ & $\begin{array}{l}\text { 09/30/92 } \\
\text { Rev. } 0 \\
\end{array}$ & TBD & Standard Waste Box & & BWHC, WMH \\
\hline $\begin{array}{l}\text { WHC-SD-TP-SARP- } \\
010 \text { Rev. } 0\end{array}$ & $\begin{array}{l}01 / 30 / 96 \\
\text { Rev. } 0 \\
\end{array}$ & 2000 & Interim Storage Cask & & DESH, BWHC \\
\hline $\begin{array}{l}\text { WHC-SD-TP-SARP- } \\
011 \text { Rev. } 0 \text {-A }\end{array}$ & $\begin{array}{l}\text { 07/28/95 } \\
\text { Rev. } 0 \\
\end{array}$ & 2000 & N-Reactor Fuel Spacers & & BHI \\
\hline $\begin{array}{l}\text { WHC-SD-TP-SARP- } \\
012 \text { Rev 1-D }\end{array}$ & $\begin{array}{l}02 / 29 / 96 \\
\text { Rev, } 1 \\
\end{array}$ & 2000 & L3-181 N Basin Cask & & BHI \\
\hline
\end{tabular}


Table 2-1. SARP Upgrade Schedule (5 pages)

\begin{tabular}{|c|c|c|c|c|c|}
\hline \multicolumn{6}{|c|}{ FY 2001 SARP Upgrades } \\
\hline $\begin{array}{l}\text { SARP Number and } \\
\text { Current Revision }\end{array}$ & $\begin{array}{l}\text { Last } \\
\text { Complete } \\
\text { Review }\end{array}$ & Expires & Title & Plan/Status & Users \\
\hline $\begin{array}{l}\text { WHC-SD-TP-SARP- } \\
001 \text { Rev. 1-B }\end{array}$ & $\begin{array}{l}11 / 15 / 96 \\
\text { Rev. } 1\end{array}$ & 2001 & Sample Pig Transport System & & $\begin{array}{l}\text { WMH, DESH, } \\
\text { BHI, NHC, } \\
\text { BWHC, LMHC }\end{array}$ \\
\hline $\begin{array}{l}\text { WHC-SD-TP-SARP- } \\
002 \text { Rev. 1-D }\end{array}$ & $\begin{array}{l}11 / 15 / 96 \\
\text { Rev. } 1\end{array}$ & 2001 & Onsite Transfer Cask System & & LMHC, NHC \\
\hline $\begin{array}{l}\text { WHC-SD-TP-SARP- } \\
008 \text { Rev. 0-B }\end{array}$ & $\begin{array}{l}09 / 26 / 94 \\
\text { Rev. } 0\end{array}$ & TBD & NRF TRIGA Packaging & & $\begin{array}{l}\text { BWHC, } \\
\text { COGEMA }\end{array}$ \\
\hline $\begin{array}{l}\text { WHC-SD-TP-SARP- } \\
009 \text { Rev, 0-A }\end{array}$ & $\begin{array}{l}08 / 15 / 96 \\
\text { Rev. } 0\end{array}$ & 2001 & LR 56/H Cask System & & $\begin{array}{l}\text { LMHC, NHC, } \\
\text { COGEMA }\end{array}$ \\
\hline $\begin{array}{l}\text { WHC-SD-TP-SARP- } \\
015 \text { Rev. 0-E }\end{array}$ & $\begin{array}{l}10 / 01 / 96 \\
\text { Rev. } 0\end{array}$ & 2001 & Double-Shell Tank Car & & WMH, DYN \\
\hline $\begin{array}{l}\text { WHC-SD-TP-SARP- } \\
016 \text { Rev. 0-B }\end{array}$ & $\begin{array}{l}02 / 23 / 96 \\
\text { Rev. } 0\end{array}$ & 2001 & Hanford Ecorok Packaging & & DESH \\
\hline $\begin{array}{l}\text { WHC-SD-TP-SARP- } \\
018 \text { Rev. 0-B }\end{array}$ & $\begin{array}{l}09 / 21 / 96 \\
\text { Rev. } 0\end{array}$ & 2001 & $\begin{array}{l}\text { Type B Quantities of } \\
\text { Radioactive Material in Type } \\
\text { A Boxes }\end{array}$ & & WMH \\
\hline \multicolumn{6}{|c|}{ FY 2002 SARP Upgrades } \\
\hline $\begin{array}{l}\text { SARP Number and } \\
\text { Current Revision }\end{array}$ & $\begin{array}{l}\text { Last } \\
\text { Complete } \\
\text { Review } \\
\end{array}$ & Expires & Title & Plan/Status & Users \\
\hline $\begin{array}{l}\text { HNF-SD-TP-SARP- } \\
021 \text { Rev. 0-B }\end{array}$ & $\begin{array}{l}04 / 02 / 97 \\
\text { Rev. } 0\end{array}$ & 2002 & Castor GSF Cask & & BWHC \\
\hline $\begin{array}{l}\text { HNF-SD-TP-SARP- } \\
022 \text { Rev. 0-B }\end{array}$ & $\begin{array}{l}03 / 17 / 97 \\
\text { Rev. } 0\end{array}$ & 2002 & GNS-12 Packaging & & BWHC \\
\hline $\begin{array}{l}\text { HNF-SD-TP-SARP- } \\
023 \text { Rev. 0-B }\end{array}$ & $\begin{array}{l}02 / 24 / 97 \\
\text { Rev. } 0\end{array}$ & 2002 & $\begin{array}{l}\text { Doorstop Sample Carrier } \\
\text { System }\end{array}$ & & $\begin{array}{l}\text { LMHC, NHC, } \\
\text { DESH, WMH, } \\
\text { BWHC, BHI }\end{array}$ \\
\hline $\begin{array}{l}\text { HNF-SD-TP-SARP- } \\
013 \text { Rev. } 0\end{array}$ & $\begin{array}{l}\text { 05/09/97 } \\
\text { Rev. } 0 \\
\end{array}$ & 2002 & $\begin{array}{l}\text { Long Length Contaminated } \\
\text { Equipment Transport System }\end{array}$ & & \\
\hline $\begin{array}{l}\text { HNF-SD-TP-SARP- } \\
020 \text { Rev. } 0\end{array}$ & $\begin{array}{l}03 / 26 / 97 \\
\text { Rev. } 0\end{array}$ & 2002 & T-3 Cask & & \\
\hline $\begin{array}{l}\text { HNF-SD-TP-SARP. } \\
017 \text { Rev. } 0\end{array}$ & $\begin{array}{l}07 / 14 / 97 \\
\text { Rev. } 0\end{array}$ & 2002 & Multicanister Overpack Cask & & \\
\hline $\begin{array}{l}\text { HNF-SD-TP-SARP- } \\
025 \text { Rev. } 0\end{array}$ & $\begin{array}{l}06 / 06 / 97 \\
\text { Rev. } 0\end{array}$ & 2002 & $\begin{array}{l}\text { Limited Type B Material in } \\
\text { CNS 14-215H Cask }\end{array}$ & & \\
\hline
\end{tabular}


Table 2-1. SARP Upgrade Schedule (5 pages)

\begin{tabular}{|c|c|c|c|c|c|}
\hline $\begin{array}{l}\text { HNF-SD-TP-SARP- } \\
026 \text { Rev. } 0\end{array}$ & $\begin{array}{l}\text { 09/25/97 } \\
\text { Rev. } 0\end{array}$ & 2002 & $\begin{array}{l}\text { Transuranic Performance } \\
\text { Demonstration Program } \\
\text { Sample Packaging }\end{array}$ & & \\
\hline $\begin{array}{l}\text { HNF-SD-TP-SARP- } \\
027 \text { Rev. } 0 \\
\end{array}$ & $\begin{array}{l}09 / 30 / 97 \\
\text { Rev. } 0 \\
\end{array}$ & 2002 & WESF Ion Exchange Module & & \\
\hline \multicolumn{6}{|c|}{ FY 2003 SARP Upgrades } \\
\hline $\begin{array}{l}\text { SARP Number and } \\
\text { Current Revision }\end{array}$ & $\begin{array}{l}\text { Last } \\
\text { Complete } \\
\text { Review }\end{array}$ & Expires & Title & Plan/Status & Users \\
\hline $\begin{array}{l}\text { HNF-334I } \\
\text { Rev. } 0\end{array}$ & $\begin{array}{l}09 / 98 \\
\text { Rev. } 0\end{array}$ & 2003 & $\begin{array}{l}\text { Decontaminated Equipment } \\
\text { Self-Container }\end{array}$ & & \\
\hline $\begin{array}{l}\text { HNF-2209 } \\
\text { Rev. } 0\end{array}$ & $\begin{array}{l}09 / 98 \\
\text { Rev. } 0\end{array}$ & 2003 & Steel Drum & & \\
\hline $\begin{array}{l}\text { HNF-2760 } \\
\text { Rev. } 0\end{array}$ & $\begin{array}{l}09 / 98 \\
\text { Rev. } 0\end{array}$ & 2003 & Ion Exchange Modules & & \\
\hline $\begin{array}{l}\text { HNF-2568 } \\
\text { Rev. } 0\end{array}$ & $\begin{array}{l}5 / 98 \\
\text { Rev. } 0\end{array}$ & 2003 & Contaminated Well Cars & & \\
\hline
\end{tabular}

\subsection{SUMMARY AND RECOMMENDATIONS}

This plan is a living document that is used as a management tool for implementing requirements in HNF-PRO-154, Responsibilities and Procedures for All Hazardous Material Shipments, and the PHMC SARP performance agreement for the FY 1997 through FY 2001 period. This plan provides the schedule for SARP upgrades and cancellations. It should be noted that the following uncertainties will affect the implementation of this plan. The plan will be changed as necessary.

- Operational requirements may lead to a SARP upgrade ahead of schedule. In those cases, other SARPs may be moved within the plan based on available funding and staffing. 
- $\quad$ Funding and workload may result in changes to this plan by management prerogative. This plan will be revisited and revised periodically until all SARPs are upgraded. This revision to the plan reflects a SARP upgrade schedule based on the most current program needs. Future revisions to the plan will include results of the plan, beginning in FY 1997.

- $\quad$ All SARPs shall be reviewed and updated as necessary at least every five years, and shall have controlled distribution as outlined in HNF-PRO-154 Responsibilities and Procedures for All Hazardous Material Shipments.

- A standardized SARP numbering system will be applied to all SARPs.

\subsection{REFERENCES}

Fisher, A. J., 1992, Westinghouse Hanford Company's Response to the RL Surveillance Numbers CMP-WFD-92-066 and CMP-WFD-92-106, (letter 9259544 to J. M. Hennig, December 23), Westinghouse Hanford Company, Richland, Washington.

HNF-PRO-154, Responsibilities and Procedures for All Hazardous Material Shipments, Fluor Daniel Hanford, Inc., Richland, Washington.

HNF-PRO-705, Safety Basis Planning, Documentation, Review and Approval, Fluor Daniel Hanford, Inc., Richland, Washington.

WHC, 1997, Safety Analysis Report for Packaging Upgrade Plan, WHC-SD-TP-PLN-006, Revision 5, Waste Management Federal Services, Inc., Northwest Operations. 


\section{DISTRIBUTION SHEET}

\begin{tabular}{|c|c|c|c|c|c|}
\hline \multirow{2}{*}{$\begin{array}{l}\text { To } \\
\text { Distribution }\end{array}$} & \multirow{2}{*}{\multicolumn{3}{|c|}{$\begin{array}{l}\text { From } \\
\text { Packaging Engineering }\end{array}$}} & \multicolumn{2}{|l|}{ Page I of 1} \\
\hline & & & & \multicolumn{2}{|c|}{ Date $10 / 27 / 98$} \\
\hline \multirow{2}{*}{\multicolumn{4}{|c|}{$\begin{array}{l}\text { Project Title/Work Order } \\
\text { Safety Analysis Report for Packaging Upgrade Plan } \\
\text { (HNF-SD-TP-PLN-006) }\end{array}$}} & \multirow{2}{*}{\multicolumn{2}{|c|}{$\begin{array}{ll}\text { EDT No. } & \text { NA } \\
\text { ECN No. } & 646738\end{array}$}} \\
\hline & & & & & \\
\hline \multicolumn{2}{|l|}{ Name } & $\begin{array}{l}\text { Text } \\
\text { With All } \\
\text { Attach. }\end{array}$ & Text Only & $\begin{array}{l}\text { Attach./ } \\
\text { Appendix } \\
\text { Only }\end{array}$ & $\begin{array}{c}\text { EDT/ECN } \\
\text { Only }\end{array}$ \\
\hline $\begin{array}{l}\text { P. K. Brockman } \\
\text { D. W. Claussen } \\
\text { J. G. Field } \\
\text { J. W. Golden } \\
\text { D. L. Kelly } \\
\text { C. G. Mattson } \\
\text { T. L. Moore } \\
\text { R. J. Smith } \\
\text { HNF-SD-TP-PLN-006 File } \\
\text { Work Control (D. L. Kelly) } \\
\text { Central Files }\end{array}$ & $\begin{array}{l}H 1-11 \\
S 7-55 \\
H 1-15 \\
N 1-26 \\
H 1-15 \\
N 1-26 \\
H 6-30 \\
H 1-15 \\
H 1-15 \\
H 1-15 \\
B 1-07\end{array}$ & $\begin{array}{l}X \\
X \\
X \\
X \\
X \\
X \\
X \\
X \\
X \\
X \\
X\end{array}$ & & & $x$ \\
\hline DOE Reading Room & H2-53 & $x$ & & & \\
\hline
\end{tabular}

